\title{
Fractional Quantum Hall States of Atoms in Optical Lattices
}

\section{Citation}

Sørensen, Anders S., Eugene Demler, and Mikhail D. Lukin. 2005. "Fractional Quantum Hall States of Atoms in Optical Lattices." Physical Review Letters 94 (8) (March 2). doi:10.1103/ physrevlett.94.086803.

\section{Published Version}

doi:10.1103/physrevlett. 94.086803

\section{Permanent link}

http://nrs.harvard.edu/urn-3:HUL.InstRepos:27899423

\section{Terms of Use}

This article was downloaded from Harvard University's DASH repository, and is made available under the terms and conditions applicable to Other Posted Material, as set forth at http:// nrs.harvard.edu/urn-3:HUL.InstRepos:dash.current.terms-of-use\#LAA

\section{Share Your Story}

The Harvard community has made this article openly available.

Please share how this access benefits you. Submit a story.

Accessibility 


\title{
Fractional Quantum Hall States of Atoms in Optical Lattices
}

\author{
Anders S. Sørensen, ${ }^{1,2,3}$ Eugene Demler, ${ }^{2}$ and Mikhail D. Lukin ${ }^{1,2}$ \\ ${ }^{1}$ ITAMP, Harvard-Smithsonian Center for Astrophysics, Harvard University, Cambridge, Massachusetts 02138, USA \\ ${ }^{2}$ Physics Department, Harvard University, Cambridge, Massachusetts 02138, USA \\ ${ }^{3}$ Niels Bohr Institute, University of Copenhagen, DK-2100 Copenhagen $\emptyset$, Denmark
}

(Received 6 May 2004; published 2 March 2005)

\begin{abstract}
We describe a method to create fractional quantum Hall states of atoms confined in optical lattices. We show that the dynamics of the atoms in the lattice is analogous to the motion of a charged particle in a magnetic field if an oscillating quadrupole potential is applied together with a periodic modulation of the tunneling between lattice sites. In a suitable parameter regime the ground state in the lattice is of the fractional quantum Hall type, and we show how these states can be reached by melting a Mott-insulator state in a superlattice potential. Finally, we discuss techniques to observe these strongly correlated states.
\end{abstract}

PACS numbers: 73.43.-f, 03.75.Lm

Ultracold atomic gases [1] provide a unique access to quantum many-body systems with well understood and controllable interactions. Whereas most of the experiments in this field have been carried out in the regime of weak interactions, the recent achievements involving Feshbach resonances [2] and the realization of a Mott-insulator state of atoms in optical lattices [3,4] enters into the regime of strong interaction with a richer and more complex manybody dynamics. At the same time, a realization of strongly correlated states of fractional quantum Hall type [5] has recently been suggested in rotating cold atomic gases [6]. However, weak interaction between the particles (and correspondingly small gap in the excitation spectrum) required precision on trap rotation, and finite temperature effects make these proposals difficult to realize experimentally. In this Letter we present a novel method that uses atoms in optical lattices to create states of the fractional quantum Hall type. Since the interactions of atoms localized in the lattices are strongly enhanced compared to the interaction of atoms in free space, these states are characterized by large energy gaps.

The fractional quantum Hall effect occurs for electrons confined to two dimensions (the $x y$ plane) in a strong magnetic field. In the simplest form the effect occurs if the number of magnetic fluxes $N_{\phi}$ is an integer $m$ times the number of particles $N_{\phi}=m N$. At this magnetic field the ground state of the system is separated from all other states by an energy gap and is well described by the Laughlin wave function [7]

$$
\Psi\left(z_{1}, z_{2}, \ldots, z_{N}\right)=e^{\left(-\sum_{j}\left|z_{j}\right|^{2} / 4\right)} \prod_{j<k}\left(z_{j}-z_{k}\right)^{m},
$$

where $z=x+i y$, and where we have assumed the symmetric gauge and suitable magnetic units. Because of the Pauli principle, only the states with odd (even) $m$ are applicable to fermions (bosons). In this Letter for simplicity we consider only bosons and $m=2$.
Below we describe a method to produce the states (1) for ultracold atoms in an optical lattice. In this system the states are protected by an excitation gap controlled by the tunneling energy, which for typical parameters is much larger than the energy scale in the magnetic traps considered previously [6]. The larger energy gap is a clear advantage experimentally because the state will be more robust to external perturbations. Furthermore, experiments with periodically modulated quantum Hall probes [8] and tunnel coupled superconducting islands [9] are trying to reach the regime studied here, and the extension of the quantum Hall physics to a lattice system is therefore an interesting nontrivial problem in its own right. For noninteracting particles the energy spectrum on the lattice (the so-called Hofstadter butterfly [10]) is very different from that of Landau levels. We nevertheless show that in a certain parameter regime, the Laughlin state provides a reasonable description for the resulting many-body physics of the strongly interacting system.

We consider atoms trapped in a square two-dimensional optical lattice. If the atoms in the lattice are restricted to the lowest Bloch band the system can be described by a BoseHubbard Hamiltonian [11]

$$
H=-J \sum_{\{j, k\}}\left(\hat{a}_{j}^{\dagger} \hat{a}_{k}+\hat{a}_{k}^{\dagger} \hat{a}_{j}\right)+U \sum_{j} n_{j}\left(n_{j}-1\right),
$$

where the first sum is over neighboring sites $j$ and $k, \hat{a}_{j}$ and $n_{j}=\hat{a}_{j}^{\dagger} \hat{a}_{j}$ are the boson annihilation and number operators on site $j, J$ is the tunneling amplitude, and $U$ is the onsite interaction energy. The individual lattice sites below are specified by a pair of integers $x$ and $y$.

An essential ingredient in our approach is an effective magnetic field for neutral atoms in optical lattices. Different approaches which attain this goal have already been proposed [12], but here we present an alternative procedure that may simplify the experimental realization. Our procedure involves a combination of a time-varying quadrupolar potential $V(t)=V_{\mathrm{qp}} \sin (\omega t) \hat{x} \hat{y}$, and a modu- 
lation of the tunneling in time. The tunneling between neighboring sites decreases exponentially with the intensity of the lasers creating the lattice, whereas the shape of the wave packet (the Wannier functions) has a much weaker dependence [11]. By varying the laser intensity, the tunneling can therefore be varied rapidly in time. Assume that the tunneling in the $x$ direction is turned on for a short period around $t=t_{0} n(n=0,1,2, \ldots)$ and that the tunneling in the $y$ direction is turned on for a short period around $t=t_{0}(n+1 / 2)$, where $t_{0}=2 \pi / \omega$ is the period of the oscillation [see Fig. 1(a)]. As illustrated by the simplified picture in Figs. 1(b) and 1(c), such a time sequence creates an effective Lorentz force (magnetic field) in the lattice. This can be shown mathematically by assuming that the tunneling is present only in a very short time interval $\tau$. With a periodic oscillation in the Hamiltonian the time evolution operator after $m$ periods can be written as a product of the evolution in each period $U\left(t=m t_{0}\right)=U\left(t=t_{0}\right)^{m}$. From the general solution to the Schrödinger equation $U=\mathcal{T} \exp \left[-i \int d t^{\prime} H\left(t^{\prime}\right) / \hbar\right]$, where $\mathcal{T}$ denotes time ordering, we find the total time evolution operator (neglecting for now the interaction between the particles)

$$
\begin{aligned}
U\left(t=\frac{m 2 \pi}{\omega}\right)= & \left(e^{-i \tau T_{x} / 2 \hbar} e^{2 i V_{\mathrm{qp}} \hat{x} \hat{y} / \omega \hbar} e^{-i \tau T_{y} / \hbar}\right. \\
& \left.\times e^{-2 i V_{\mathrm{qp}} \hat{x} \hat{y} / \omega \hbar} e^{-i \tau T_{x} / 2 \hbar}\right)^{m},
\end{aligned}
$$

where $T_{x}$ and $T_{y}$ are the kinetic energy operators describing (a)

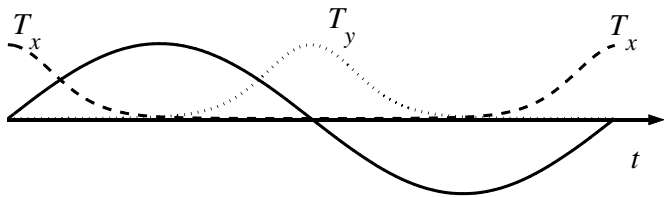

(b)

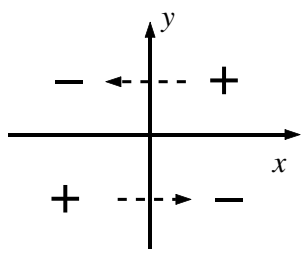

(c)

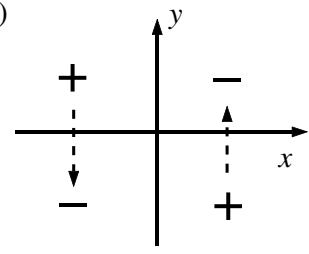

FIG. 1. (a) One period of the sequence used to create an effective magnetic field. The time evolution of the quadrupole potential is shown by the full curve, and the dashed and the dotted lines indicate the tunneling in the $x$ and the $y$ directions. For illustration the shown evolution of the tunneling is obtained from a sinusoidal variation of the lattice potential between 5 and 40 recoil energies [11]. (b),(c) Physical explanation of the procedure. (b) Tunneling in the $x$ direction is followed by a positive potential in the first and third quadrant (signs in the figure), and hence atoms will experience a lower potential by moving in the direction of the dashed arrows. (c) Same as (b) but with tunneling in the $y$ direction and opposite sign of the potential. When combined, the dashed lines in (b) and (c) make a circular cyclotron motion. tunneling in the $x$ and $y$ directions, respectively, e.g., $T_{x}=$ $-J \sum \hat{a}_{x+1, y}^{\dagger} \hat{a}_{x, y}+$ H.c. $\quad$ By using $\exp (i 2 \pi \alpha \hat{x} \hat{y}) \times$ $\hat{a}_{x, y+1}^{\dagger} \hat{a}_{x, y} \exp (-i 2 \pi \alpha \hat{x} \hat{y})=\hat{a}_{x, y+1}^{\dagger} \hat{a}_{x, y} \exp (i 2 \pi \alpha x)$ with $\alpha=V_{\mathrm{qp}} / \pi \hbar \omega$, (3) can be reduced to the time evolution $U=\exp \left(-i H_{\mathrm{eff}} t / \hbar\right)$ from an effective Hamiltonian

$$
H_{\mathrm{eff}} \approx-J \sum_{x, y} \hat{a}_{x+1, y}^{\dagger} \hat{a}_{x, y}+\hat{a}_{x, y+1}^{\dagger} \hat{a}_{x, y} e^{i 2 \pi \alpha x}+\text { H.c., }
$$

where the effective tunneling strength is $J=J \tau / t_{0}$, and where we have omitted terms of order $J(J / \omega)^{2}$. Equation (4) describes the behavior of a charged particle on a lattice with a magnetic flux $\alpha \Phi_{0}$ going through each unit cell $[10,13]$, and hence the procedure introduces an effective magnetic field in the lattice. The gauge in Eq. (4) (Landau gauge) is determined by the time we terminate the sequence in Fig. 1(a) and a different gauge would appear if we terminated at a different time.

We now turn to the fractional quantum Hall effect for strongly interacting atoms. In the limit of small $\alpha$ and a small number of atoms per lattice site the system reduces to the continuum limit of particles in a magnetic field with short range interactions. If the interaction is repulsive, the Laughlin wave function (1) is the absolute ground state of the system when $N_{\phi}=m N$ [14]. This limit corresponds to a very low density gas with a small energy gap between the ground and the excited states (see below). To extend this analysis to nonvanishing $\alpha$ we have performed a direct numerical diagonalization of the Hamiltonian in Eq. (4) for a small number of hard-core bosons; i.e., corresponding to the limit $J \ll U$, we diagonalize the Hamiltonian in Eq. (4) excluding from the Hilbert space all states with more than one boson at each site. In different contexts, similar problems were considered in Ref. [15].

To investigate the effect of finite $\alpha$ we fix the number of fluxes and particles so that $N_{\phi}=2 N$ and vary $\alpha$ by changing the size of the lattice. (The sizes in the $x$ and the $y$ directions are the same or different by unity.) In Fig. 2(a) we show the overlap of the ground state wave function
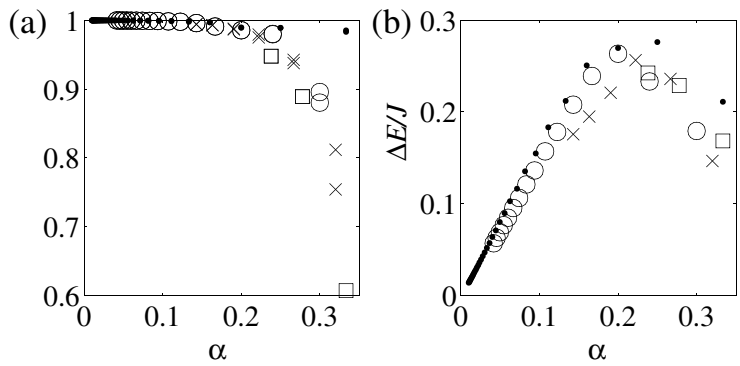

FIG. 2. (a) Overlap of the ground state wave function with the Laughlin wave function (1). (b) Energy gap $\Delta E$ to the lowest excited state. In the figure we have fixed the number of particles and fluxes $\left(N_{\phi}=2 N\right)$ and vary the flux per unit cell $\alpha$ by varying the size of the lattice. The shown results are for $N=$ $2(\bigcirc), N=3(\bigcirc), N=4(\times)$, and $N=5(\square)$. 
from the diagonalization with the Laughlin wave function (1). We assume periodic boundary conditions to represent the bulk properties of a large optical lattice. For the situation considered here $(m=2)$ the combination of periodic boundary conditions and magnetic field gives rise to a twofold degeneracy of the ground state in the continuum limit ( $\alpha \ll 1)$, where the two ground states differ only by their center of mass wave functions [16]. The symmetry analysis leading to this degeneracy does not apply in the presence of the lattice [17]. For all points in Fig. 2 (except the point $N=5, \alpha=1 / 3$ ), however, the diagonalization gives two almost degenerate ground states which are separated from the excited states [see Fig. 3(a) at $V_{0}=0$ ]. The periodic generalization of the Laughlin wave function [18] also has a twofold center of mass degeneracy, and in Fig. 2(a) we show the overlap of the two lowest states from the diagonalization with the subspace spanned by the Laughlin wave functions.

Our simulations show a very good overlap with the Laughlin wave function for $\alpha \lesssim 0.3$, but the overlap starts to fall off for $\alpha \geqslant 0.3$. The excellent overlap with the Laughlin wave function is not accidental; e.g., for $N=5$ and $\alpha \approx 0.24$ the size of the Hilbert space is $8.5 \times 10^{5}$ and the overlap is $95 \%$. These numerical calculations therefore provide strong evidence that the Laughlin wave function captures the essential properties of the many-particle system. Experimentally, it is desirable to have a large excitation gap. From the results in Fig. 2(b) we see that the optimal regime is $\alpha \sim 0.2$ (although the data still have finite size effects). In this region the Laughlin wave function (1) is a very good description of the state.
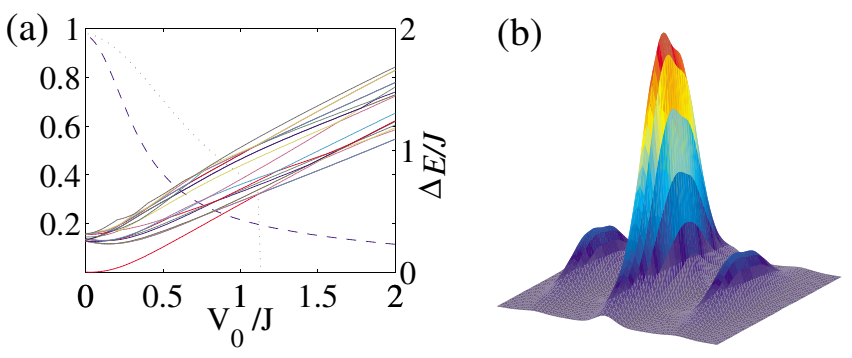

FIG. 3 (color online). (a) Creation of the Laughlin state by melting a Mott insulator. Full lines (right axis): energy relative to the ground state of the 19 lowest excited states for different amplitudes $\left(V_{0}\right)$ of the superlattice potential. Dashed (dotted) lines: overlap of the ground state (first excited state) with the Laughlin wave function (1) (left axis). Note that at $V_{0}=0$ there are two nearly degenerate states because of the combination of magnetic field and periodic boundary conditions. These two states have a $98 \%$ overlap with the two possible Laughlin states. Results are for a $6 \times 6$ lattice with four atoms and eight fluxes, $\alpha \approx 0.22$. The periods of the superlattice are $p_{x}=p_{y}=3$. (b) Density distribution after expansion from the lattice (arbitrary units). For illustration we have assumed a lattice depth of ten recoil energies [11] and $\alpha \approx 0.22$ as in (a).
To reach the Laughlin state experimentally it is necessary to adiabatically load a cold Bose-Einstein condensate (BEC) into the lattice. We expect the transition between the BEC and fractional quantum Hall states to be a direct first order phase transition or proceed via several intermediate phases [19]. Thus, a direct transition between the BEC and the fractional quantum Hall state is likely to create many excitations. Instead, we suggest to enter the Laughlin state through a Mott-insulator state $[3,4,11]$. We employ an additional superlattice $V=V_{0}\left[\sin ^{2}\left(\pi x / p_{x}\right)+\right.$ $\sin ^{2}\left(\pi y / p_{y}\right)$ ], as realized experimentally in Ref. [20]. By loading a BEC into the combined potential, it is then possible to reach a Mott-insulator state with a single atom at each of the potential minima of the superlattice $[3,4,11]$. In this state the atoms are unaffected by the turnon of the effective magnetic field. By reducing the superlattice potential, it is then possible to adiabatically reach the Laughlin state. In Fig. 3(a) we show the evolution of the lowest energy levels when we reduce the superlattice potential $V_{0}$. For $V_{0} \gg J$ the ground state is the Mottinsulator state which is well separated from all excited states. When $V_{0}=0$, there are two nearly degenerate ground states because of the center of mass degeneracy mentioned above. Apart from this artifact of the periodic boundary conditions, the ground state is always well separated from the excited states by an energy gap, and the ground state of the system smoothly changes into the Laughlin state, so that it is possible to reach the Laughlin state by slowly reducing the superlattice potential.

We next consider experimental issues involving the realization and detection of quantum Hall states. Under realistic conditions the required "hard-core" limit can be reached, e.g., in atomic $\mathrm{Rb}$ with a tunneling rate $J / 2 \pi \hbar$ of the order of hundreds of $\mathrm{Hz}[3,11]$, which indicates that energy gaps in the range $10-100 \mathrm{~Hz}$ can be obtained. To observe the quantum Hall states, the temperature needs to be below this excitation gap which requires realistic temperatures of a few $\mathrm{nK}$. A limitation is that the oscillating potentials produce strong phase shifts on the atoms. If there is a total of $N_{\phi}$ fluxes in the lattice, the phase shift on atoms in the outermost regions is on the order of $N_{\phi}$ per halfcycle. Hence a practical implementation requires strong gradients, and the phase shift in one-half of the pulse must exactly balance the phase shift in the other. Another limitation is that the oscillating quadrupole potential can excite higher Bloch bands. If we approximate the wells by a harmonic potential, the weight on the excited state in the outermost regions of the lattice is $w_{\mathrm{ex}} \sim$ $\pi N_{\phi} \alpha\left(a_{0} / \lambda\right)^{2} \omega^{2} / \nu_{b}^{2}$, where $\nu_{b}$ is the Bloch-band separation, and where the ground state width $a_{0}$ is of order $\lambda / 10$ for typical parameters [11]. With $\omega \sim \nu_{b} / 10$ this is not a major concern for $N_{\phi} \lesssim 10^{2}-10^{3}$.

To demonstrate experimentally that one has reached the Laughlin states one would ideally probe some of its unique features, such as incompressibility, the fractional charge of 
the excitations, or their anyonic character. Such probes will most likely be very challenging to implement and we shall now discuss a simpler experimental indication. In most experiments with cold trapped atoms the state is probed by releasing the atoms from the trap and imaging the momentum distribution. To show that such an expansion gives useful information we use the continuum wave function (1) which is a good description in the regime we are interested in. In the lowest Landau level the single particle density matrix for any state with constant density was found in Ref. [21]. From this density matrix we find the asymmetric expansion shown in Fig. 3(b). This density distribution is clearly distinct from, e.g., a superfluid state which will have Bragg peaks, and a Mott-insulator, which gives a symmetric distribution [3]. This method, however, does not reveal detailed information about the state except that it is in the lowest Landau level. Further insight can be obtained by measuring higher order correlation functions $[22,23]$, by probing the excitation spectrum through stimulated Bragg scattering [24], or by studying edge states [25] (note that the latter does not appear in our numerics because of the periodic boundary conditions).

To summarize, we have presented a feasible method to construct fractional quantum Hall states in an optical lattice. Compared to previous proposals with cold atoms [6] the optical lattice approach results in a more robust quantum Hall state, since it is protected by a larger gap. The present approach therefore reduces the experimental requirement and could facilitate the observation of such states with a larger number of particles.

Several interesting new avenues are opened by this work. First of all, it would be interesting to understand the exact nature of the ground state when there is a large flux fraction per unit cell $\alpha \gtrsim 0.3$. We have not made any modification to the Laughlin wave function to take the lattice into account, and one would expect this to reduce the overlap in Fig. 2(a) when $\alpha$ is not vanishingly small. The decrease in the overlap is, however, much less abrupt for the single particle wave functions. We therefore do not expect this to explain the observed results. Alternatively, the decrease could be caused by the system entering a different phase for $\alpha \gtrsim 0.3$, e.g., a superfluid state with a vortex lattice. This possibility is supported by the decrease in the excitation gap above $\alpha \gtrsim 0.25$; see Fig. 2(b). We further observe an increase in the largest eigenvalue of the one-particle density matrix above $\alpha \gtrsim 0.3$ (especially for $\alpha=1 / 3$ and $1 / 2$ that correspond to a commensurate density of vortices) which is consistent with a superfluid state. We cannot, however, draw any definite conclusion from our present numerical results. In addition, the present method for creating the quantum Hall state in the lattice can easily be extended to yield different magnetic field for different internal atomic states of multicomponent bosons. Using this approach, effective non-Abelian gauge fields can be created. Therefore the present method may allow one to explore experimentally the novel properties of a many-particle system in the presence of such a field.

We are grateful for useful discussion with E. Altman, M. Greiter, B. I. Halperin, J. Taylor, P. Zoller, and the Quantum optics group at ETH, Zürich. This work was supported by NSF through Grants No. PHY-0134776 and No. DMR-0132874 and through the grant to ITAMP, by the Danish Natural Science Research Council, and by the Sloan and Packard foundations.

[1] J. R. Anglin and W. Ketterle, Nature (London) 416, 211 (2002).

[2] C. A. Regal, M. Greiner, and D. S. Jin, Phys. Rev. Lett. 92, 040403 (2004); M. W. Zwierlein et al., ibid. 92, 120403 (2004); M. Bartenstein et al., ibid. 92, 120401 (2004); J. Kinast et al., ibid. 92, 150402 (2004); T. Bourdel et al., ibid. 93, 050401 (2004).

[3] M. Greiner, O. Mandel, T. Esslinger, T. W. Hänsch, and I. Bloch, Nature (London) 415, 39 (2002).

[4] T. Stöferle et al., Phys. Rev. Lett. 92, 130403 (2004).

[5] The Quantum Hall Effect, edited by R. E. Prange and S. M. Girvin (Springer-Verlag, New York, 1990).

[6] N. K. Wilkin and J. M. F. Gunn, Phys. Rev. Lett. 84, 6 (2000); T.-L. Ho, ibid. 87, 060403 (2001); B. Paredes, P. Fedichev, J.I. Cirac, and P. Zoller, ibid. 87, 010402 (2001).

[7] R. B. Laughlin, Phys. Rev. Lett. 50, 1395 (1983).

[8] M. C. Geisler et al., Phys. Rev. Lett. 92, 256801 (2004).

[9] H.S. J. van der Zant et al., Phys. Rev. Lett. 69, 2971 (1992); B. Pannetier et al., ibid. 53, 1845 (1984).

[10] D. R. Hofstadter, Phys. Rev. B 14, 2239 (1976).

[11] D. Jaksch, C. Bruder, J. I. Cirac, C. W. Gardiner, and P. Zoller, Phys. Rev. Lett. 81, 3108 (1998).

[12] D. Jaksch and P. Zoller, New J. Phys. 5, 56 (2003); E. J. Mueller, Phys. Rev. A 70, 041603 (2004).

[13] D. Langbein, Phys. Rev. 180, 633 (1969).

[14] F. D. M. Haldane, Phys. Rev. Lett. 51, 605 (1983).

[15] G. S. Canright, S. M. Girvin, and A. Brass, Phys. Rev. Lett. 63, 2291 (1989); M. Niemeyer, J. K. Freericks, and H. Monien, Phys. Rev. B 60, 2357 (1999).

[16] F. D. M. Haldane, Phys. Rev. Lett. 55, 2095 (1985).

[17] A. Kol and N. Read, Phys. Rev. B 48, 8890 (1993).

[18] F. D. M. Haldane and E. H. Rezayi, Phys. Rev. B 31, 2529 (1985).

[19] N. Read, Phys. Rev. Lett. 65, 1502 (1990); S. Kivelson, D. H. Lee, and S. C. Zhang, Phys. Rev. B 46, 2223 (1992); X. G. Wen, Adv. Phys. 44, 405 (1995).

[20] S. Peil et al., Phys. Rev. A 67, 051603 (2003).

[21] A. H. MacDonald and S. M. Girvin, Phys. Rev. B 38, 6295 (1988).

[22] N. Read and N.R. Cooper, Phys. Rev. A 68, 035601 (2003).

[23] E. Altman, E. Demler, and M. D. Lukin, Phys. Rev. A 70, 013603 (2004).

[24] J. Stenger et al., Phys. Rev. Lett. 82, 4569 (1999).

[25] M. A. Cazalilla, N. Barberán, and N. R. Cooper, cond-mat/ 0408540. 